\title{
Update and review on the basics of brachial plexus imaging
}

\author{
Abdullah M. Addar and Ahmed A. Al-Sayed \\ *Correspondence: aalsayed89@hotmail.com

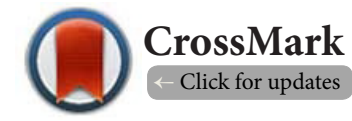 \\ Department of Surgery, College of Medicine, King Saud University, Riyadh, Saudi Arabia.
}

\begin{abstract}
Brachial plexopathy is a type of peripheral neuropathy. Injuries to the brachial plexus can be classified according to their severity, ranging from neuropraxia, the mildest form, to axonotmesis and neurotmesis, the most severe forms. The causes of brachial plexopathy include traumatic and non-traumatic injuries. Because the brachial plexus can sustain various types of injuries, different imaging modalities are required. Recent advances in diagnostic imaging have enabled better investigation of brachial plexopathy. This article reviews the major and most widely used imaging methods used for investigating brachial plexopathy along with newer modalities. The indications, advantages, and disadvantages of each modality are examined. The major factor in realizing the full potential of any imaging method is the knowledge of the requesting physician about the capabilities and limitations of each method. Magnetic resonance imaging (MRI) is the standard imaging modality for evaluating non-traumatic injury to the brachial plexus; however, there are several limitations to its use and, therefore, other modalities should be pursued. MR myelography should be used for traumatic meningoceles and root avulsions. MR neurography is a relatively new technique with massive potential. It is a tissue-specific modality with the ability to elicit morphological as well as pathological features of nerves. CT myelography is the gold standard for evaluating traumatic injury of the brachial plexus. Other potential uses are with tumors of the brachial plexus as well as obstetric brachial plexus palsies. Finally, sonography is addressed. With its ability to detect almost all plexopathies and the fact that it does not employ radiation and can be done in virtually every patient, it should be the baseline or, at least, the screening method for plexopathies.
\end{abstract}

Keywords: Brachial plexopathy, computed tomography, magnetic resonance imaging, sonography, myelography, neurography, root avulsion, peripheral nerves, lesions

\section{Introduction}

Brachial plexopathy is a form of peripheral neuropathy that may be involved in different pathological processes. The causes of brachial plexus dysfunction include traumatic injuries, which affect $1.2 \%$ of adults and $0.1 \%$ of the pediatric population [1-2], and obstetrical injuries, which occur with an incidence of 0.13 to 5.1 per 1000 live births and account for $5 \%$ of birth injuries [3].

Tumors of the brachial plexus are rare, but they can cause brachial plexopathy [4]. They can be classified as benign or malignant tumors, and each tumor type can further be categorized according to whether they are neurogenic or non-neurogenic in origin [5]. In addition, brachial plexopathy may result from tumor infiltration, compression from adjacent anatomical structures, radiation therapy [6-7], and infections or other inflammatory process [8-9].

Thoracic outlet syndrome (TOS) is a broad term that refers to entrapment and compression of the brachial plexus and other vascular structures, such as the subclavian artery and vein. It occurs in the area between the first rib and the clavicle and can be caused by several factors, such as a cervical rib, an elongated $\mathrm{C} 7$ transverse process along with nerve irritation, compression, and traction [10].

The wide spectrum of brachial plexopathies entails the application of different diagnostic modalities, with the recent advances in science and diagnostic imaging offering several options to investigate its causes. This article is aimed at reviewing the major imaging modalities of the brachial plexus, including magnetic resonance imaging (MRI), magnetic resonance myelography (MRM), computed tomography with myelography (CTM), magnetic resonance neurography (MRN), and sonography. The indications, advantages, and disadvantages of each modality are reviewed.

\section{Review}

\section{Anatomy and physiology of the brachial plexus}

To fully understand the different pathological processes involving the brachial plexus, it is necessary to have a good understanding of the developmental, structural, and functional anatomy of the brachial plexus and peripheral nerves. The peripheral nervous system develops from the neural crest cells, which form the dorsal nerve roots (sensory), and from the cells in the basal plates of the developing spinal cord, which form the ventral nerve roots (motor). Both nerve roots unite to form the mixed spinal nerve root that immediately divides into the dorsal and ventral primary rami [11]. The union of the ventral primary rami of the fifth, sixth, seventh, and eighth cervical and the first thoracic spinal nerves form the brachial plexus. The plexus can be further divided into trunks, divisions, cords, branches, and terminal branches (Figure 1).

The fifth, sixth, and seventh cervical nerve roots give rise to the long thoracic nerve. In addition, the dorsal scapular nerve is a branch of the fifth cervical nerve root. The brachial plexus 
Addar et al. Medical Imaging and Radiology 2014,

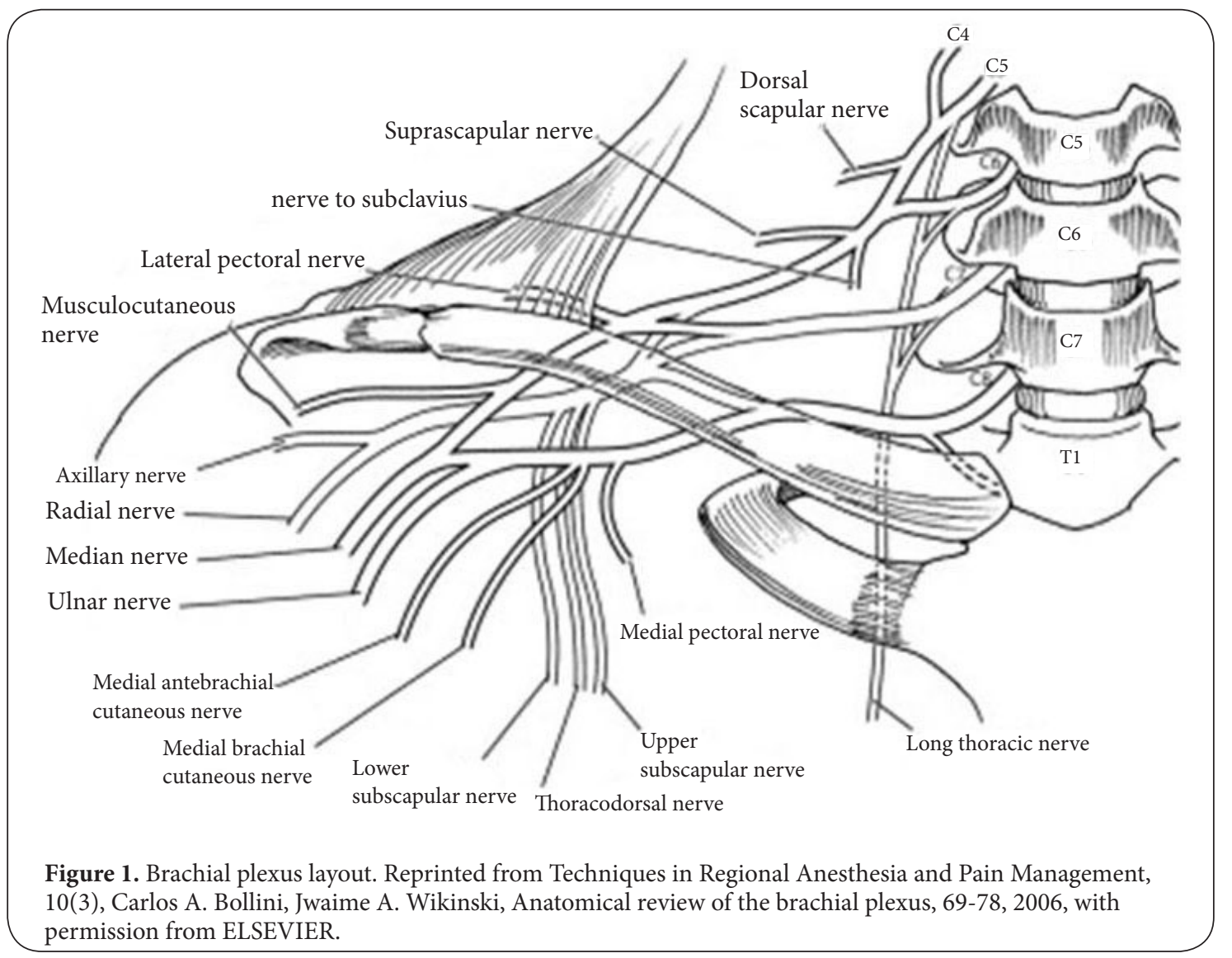

has three trunks, the upper (C5-6), the middle (C7), and the lower trunk (C8-T1), which are formed by the union of the fifth and sixth cervical nerve roots, the continuation of the seventh, and the union of the eighth cervical nerve root with the first thoracic nerve root, respectively. The upper trunk gives rise to two branches: the nerve to the subclavius and the supra-scapular nerve. Each of the three trunks divides into anterior and posterior divisions. All the posterior divisions of the upper, middle, and lower trunks unite to form the posterior cord. The posterior cord gives rise to the upper and lower subscapular nerves as well as the thoracodorsal, axillary, and radial nerves. The medial cord of the brachial plexus is a continuation of the anterior division of the lower trunk. Its branches are the medial pectoral nerve, the medial cutaneous nerve of the arm, the medial cutaneous nerve of the forearm, the ulnar nerve, and the medial root of the median nerve. The anterior divisions of the upper and middle trunks unite to form the lateral cord, which is the origin of the lateral pectoral nerve, the musculocutaneous nerve, and the lateral root of the median nerve. The cords of the brachial plexus are named according to their anatomical relation to the axillary artery; thus, they and most of their branches are present in the axilla and continue downward as terminal branches, which are the major peripheral nerves of the upper limb [12]. The complicated arrangement and efficient distribution of the brachial plexus and the peripheral nerves enable the following very important functions in the upper limb: sensory innervation, motor innervations, and sympathetic vasomotor and secretomotor nerve supply. These functions are often compromised in brachial plexopathy, which occurs at the level of the roots, trunks, or divisions that occur in the neck. Complete lesions involving all the roots of the plexus are rare and result in a flail limb, which is manifested as a complete palsy affecting the whole limb. Incomplete lesions are common and can involve either the upper part (Erb-Duchenne Palsy) or lower part (Klumpke's Palsy) of the plexus [13]. The features of each type of palsy are described in (Table 1).

Table 1. Features of each type of palsy.

\begin{tabular}{lll}
\hline & Erb-Duchenne Palsy & Klumpke's Palsy \\
\hline Description and features: & Injury to C5, C6, & Injury to C8 and \\
& and C7: & T1: \\
& C5: Patient is & C8: Patient is \\
& unable to abduct and & unable to clench \\
& externally rotate the & the fist. \\
& arm. & T1: Intrinsic \\
& C6: Patient is unable & muscles of the \\
& to flex the elbow & hand are para- \\
& joint. & lyzed. \\
& C7: Patient is unable & \\
& to extend the wrist & \\
& joint. & \\
\hline
\end{tabular}


Addar et al. Medical Imaging and Radiology 2014,

The brachial plexus, similar to the peripheral nerves, is subject to Seddon's classification of nerve injuries, which grades injuries on the basis of severity into neuropraxia, axonotmesis, and neurotmesis. Neuropraxia is the mildest form of injury, where the nerve is grossly and histologically intact but nerve conduction is interrupted physiologically. In axonotmesis, the nerve is histologically compromised; the axon and its myelin covering (endoneurium) loose continuity with the cell body; and the surrounding connective tissue framework (the epineurium and perineurium) is preserved. Neurotmesis is gross complete severance of the nerve fiber and is the most severe form (Figure 2) [14].

Injuries can also be classified to pre- and post-ganglionic injuries. Pre-ganglionic injuries are injuries in which the spinal roots are avulsed from the spinal cord, while post-ganglionic injuries are those that occur distal to the dorsal root ganglion. Pre-ganglionic injuries are characterized by the loss of motwor function only. In contrast, post-ganglionic injuries are characterizedby the loss of both sensory and motor functions.
Traditional magnetic resonance imaging (MRI) of the brachial plexus

MRI is the standard imaging modality for evaluating nontraumatic brachial plexopathies (Figure 3).

This is mainly because MRI affords multi-planar images with excellent soft-tissue contrast that is superior to that afforded by both computed tomography (CT) and sonography. It also enables the differentiation between pre- and post-ganglionic injuries, which dictates the type of treatment the patient receives. In addition, MRI is non-invasive and does not employ radiation, unlike CTM [15-16]. The diagnostic accuracy of MRI is relatively high. The overall accuracy was shown to be $87.8 \%$, with the accuracy being $93.3 \%$ for mass lesions, $87.2 \%$ for traumatic brachial plexus injuries, $83.3 \%$ for entrapment syndrome, and $83.7 \%$ for post-treatment evaluation [17]. Brachial plexus MRI is best preformed with the patients in the supine position and their arms placed at the side, by using a multi-element, phased-array radiofrequency receiver coil. The region to be imaged spans the neck to the shoulder

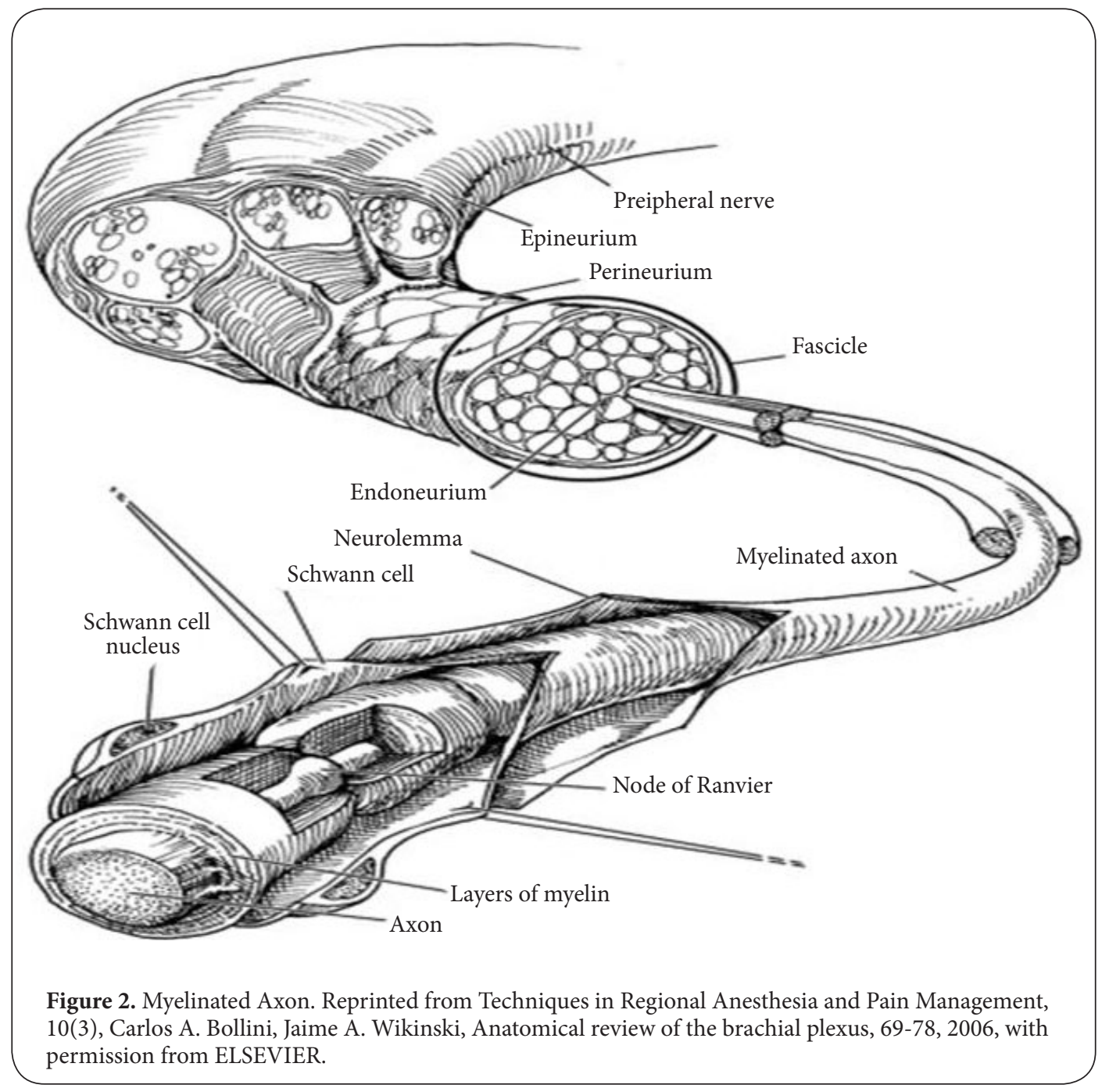




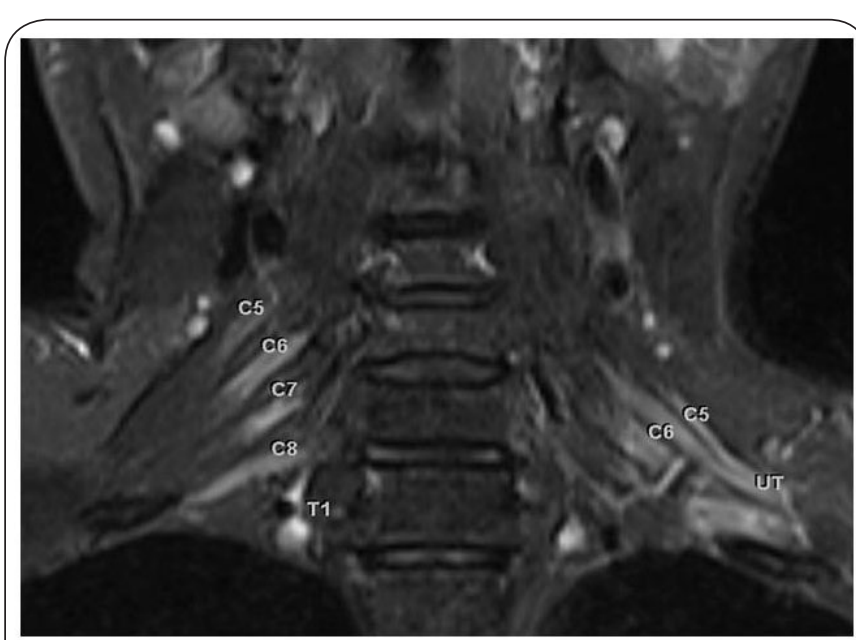

Figure 3. Normal M.R.I of the brachial plexus. T2 STIR coronal image shows the long axis of the nerve plexus as uniform mildly hyperintense fascicles, interspersed with hypointense fibrofatty connective tissue. Normal (C5, C6, C7, $\mathrm{C} 8$, and $\mathrm{T} 1$ ) brachial plexus roots/rami are seen on the right side; on the left, the upper trunk (UT) formed by C5 and C6 roots is seen. Reprinted from Clinical Radiology 64(2), J. Sureka, R.A. Cherian, M. Alexander, B.P. Thomas, MRI of brachial plexopathies, 208-218, 2009 with permission from ELSEVIER.

area, including the spine. Patients are asked to refrain from coughing and rigorous swallowing during imaging, and the images are obtained during quite respiration. Pain medications should be considered prior to image acquisition in patients suffering from plexus-related pain, to avoid motion during imaging [15-16].

MRI is utilized in traumatic brachial plexus injuries (Figure 4), where in it enables the differentiation between pre- and postganglionic injuries, although CTM and MRM are much better in detecting these lesions [15-16,18-19].

T2-weighted imaging in pre-ganglionic injuries revealssignal intensity changes in the spinal cord, nerve roots, and paraspinal muscles. For the spinal cord, T2-weighted images show hyperintense are as that might indicate edema in the acute phase and myelomalacia in the chronic phase, or hypointense areas that indicate hemorrhage with subsequent hemosiderin deposition. Enhanced nerve roots in traumatic pre-ganglionic injuries despite morphologic continuity on T1-weighted images suggest functional impairment. In addition, denervated paraspinal muscles show enhancement as early as 24 hours after nerve injury, and this is, therefore, an indirect but accurate sign of nerve root avulsion injury. In post-ganglionic injuries, thickening of the plexus reflects edema and fibrosis [18].

Primary neurogenic neoplasms, such as schwannomas, neurofibromas, neurofibrosarcomas, neuromas, and neuroblastomas, are all detectible by MRI (Figure 5).

A major disadvantage of MRI is its inability to fully and

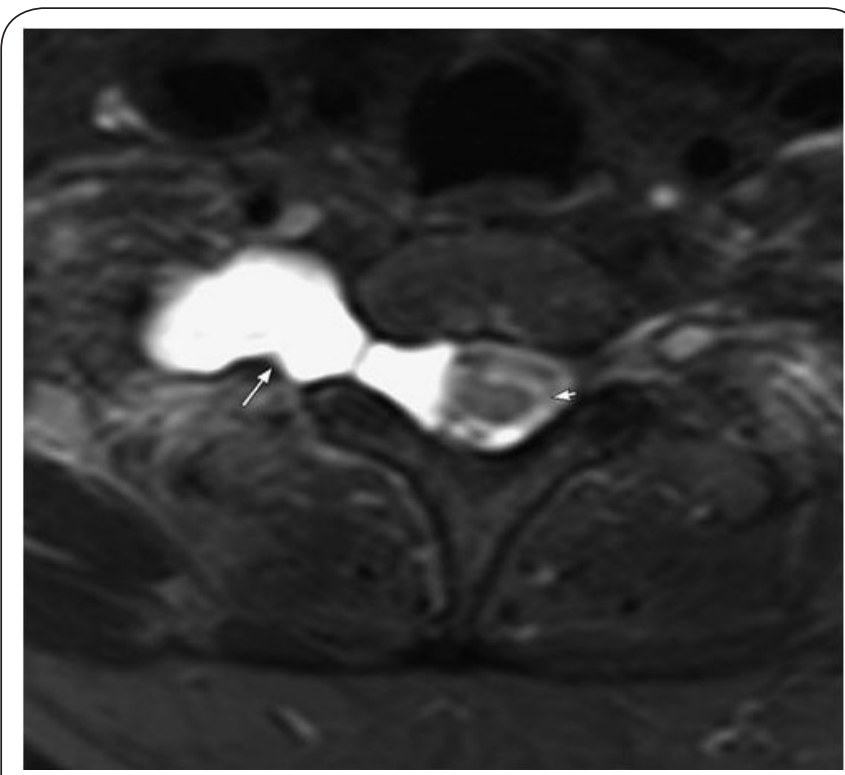

Figure 4. Preganglionic root avulsion injury with extradural large pseudomeningocele. Axial T2 STIR image shows nonvisualization of the right C8 nerve root and an associated pseudomeningocele (white arrow), which extends upto the dorsal root ganglion level. There is mild contralateral displacement of spinal cord. Intact left preganglionic nerve roots (small white arrow). Reprinted from Clinical Radiology 64(2), J. Sureka, R.A. Cherian, M. Alexander, B.P. Thomas, MRI of brachial plexopathies, 208-218, 2009 with permission from ELSEVIER.

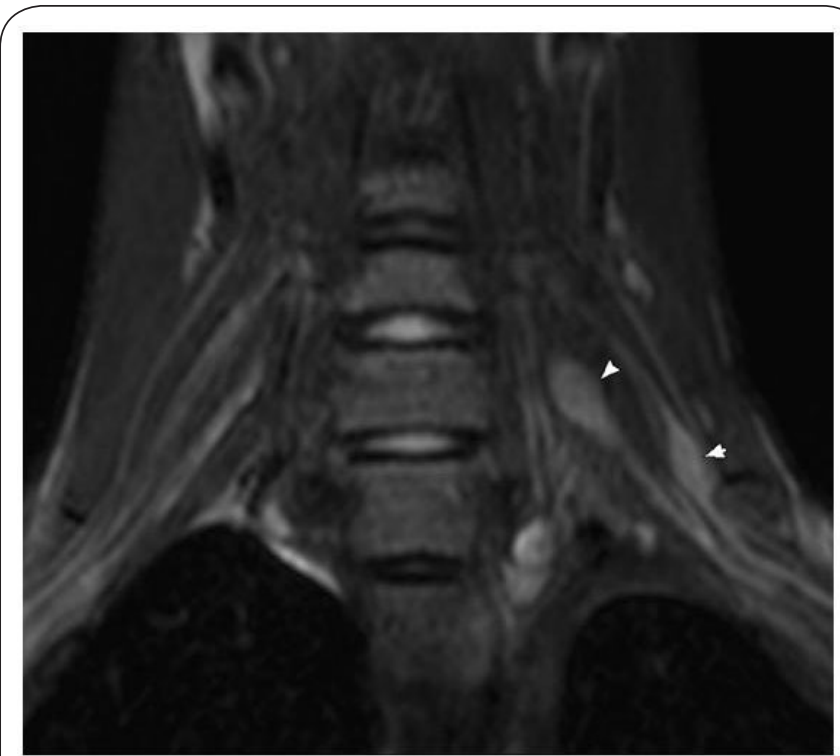

Figure 5. Neurofibroma of left C5 and C6 nerve roots/rami. T2 STIR coronal shows focal fusiform enlargement of left C5 and C6 nerve roots (white arrow heads), as compared to the normal right side. Reprinted from Clinical Radiology 64(2), J. Sureka, R.A. Cherian, M. Alexander, B.P. Thomas, MRI of brachial plexopathies, 208-218, 2009 with permission from ELSEVIER. 
reliably differentiate neurofibromas from schwannomas since their features overlap. Many signs that can differentiate a benign from a malignant tumor have been described, yet the distinction continues to be difficult and unreliable [16,20-22]. For the evaluation of metastatic neoplasms and adjacent areas, MRI has the ability to distinguish tumors that are invading the plexus from those that are adjacent to it. The brachial plexus is most commonly affected by breast cancer metastasis. Lung cancer metastatic lesions, lymphoma, melanoma, squamous cell carcinomas of the head and neck as well as the adjacent Pancoast tumor are all examples of tumors likely to affect the brachial plexus. Similar to the case with primary neoplasms of the plexus, distinguishing these neoplasms from each other is difficult through MRI since their features are nonspecific [21-22].

MRI is considerably beneficial in radiation plexopathy. In a series of 105 patients with radiation fibrosis as the most common non-traumatic pathology to affect the plexus [22], MRI could differentiate post-radiation fibrosis from recurrent or residual disease. Understandably, this differentiation, although difficult, is absolutely essential because the management of the patient would differ dramatically [21-22].

In addition, MRI is used in the diagnosis of entrapment syndrome (also known as thoracic outlet syndrome) and in the identification of its various causes, namely, hereditary motor sensory neuropathy (also known as Charcot Marie Tooth syndrome) and infective or inflammatory conditions of the brachial plexus such as brachial neuritis, chronic inflammatory demyelinating neuropathy, and other chronic infections such as leprosy and syphilis [21-22].

Despite having many uses and benefits, MRI also has several drawbacks, which must be kept in mind to optimize the use of MRI for the patients' benefit. As mentioned above, a definitive distinction between benign and malignant tumors is not yet possible. MRI has been shown to be less accurate in detecting nerve root avulsions compared to CTM and MRM [23-24]. False-positive and false-negative results may occur with MRI, especially when a careful selection of patients requiring MRI has not occurred; this highlights the importance of thereferring physician being well aware of the indications and limitations of MRI [17]. In addition to all this, MRI is time consuming, expensive, and not universally applicable to all patients; for example, MRI may not be applicable to patients with metal devices, children, and claustrophobic patients who may require general anesthesia.

\section{Magnetic resonance myelography (MRM)}

MRM is the imaging method that achieves myelogram-like images with MRI (Figure 6).

This method is rising in popularity greatly to the extent that it has started to limit the use of CTM only to patients who cannot undergo MRI or when MRI is inconclusive [15]. Its use is mainly in the diagnosis of traumatic meningoceles and nerve root avulsion, where MRM was found to be superior

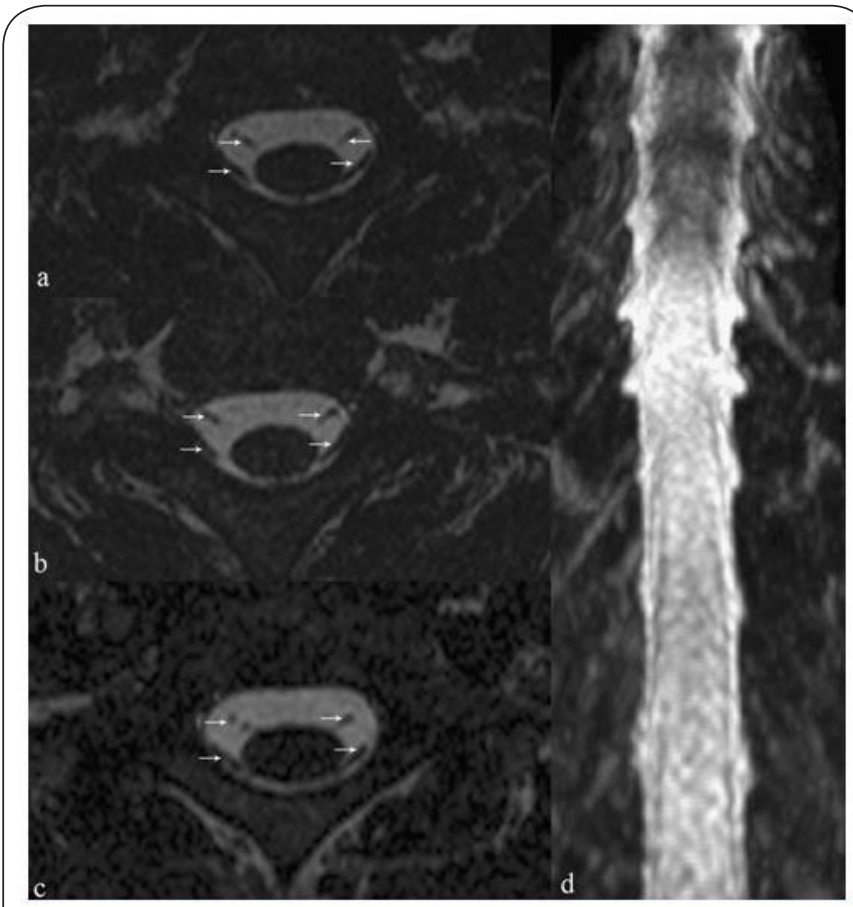

Figure 6. (a-d) Axial and 3D reconstruction of a 3D T2 MR myelography image showing excellent visualization of intradural nerve roots (arrows). CSF is depicted with a very high signal intensity, whereas the bony structures and the nerve roots have a very low signal intensity. Reprinted from European Journal of Radiology 74(2), M.I. Vargas, M. Viallon, D. Nguyen, J.Y. Beaulieu, J. Delavelle, M. Becker, New approaches in imaging of the brachial plexus, 403-410, 2010, with permission from ELSEVIER.

to CTM [15,18-19]. In addition, MRM is non-invasive, does not employ radiation, and is superior in the assessment of psuedomeningoceles compared to CTM.

The rationale for using MRM is mainly that the diagnostic accuracy of traditional MRI in detecting root avulsions is $52 \%$ and that of CTM is $85 \%$ [23], while MRM is superior to CTM, with a diagnostic accuracy of $92 \%$ [19]. In addition, MRM can be employed in the acute phase of injury unlike CTM, where lumbar puncture and use of contrast media carries a slight risk [25].

The radiographic morphological features of the pre-ganglionic lesions detected by MRM are as follows: (1) signal changes in the spinal cord, (2) hemorrhage near the nerve root exit, (3) no visualization of the nerve roots, (4) discontinuity of the nerve roots, (5) cerebrospinal fluid(CSF) leakage, (6) psuedomeningoceles, and (7) enhancement of paraspinal muscles [23].

Again, despite the superior qualities of MRM, it has some limitations that the referring physician must be aware of. First, MRM images may contain CSF flow artifacts. CSF flows in a pulsatile fashion, and neglecting to account for CSF flow may lead to a false-positive diagnosis of a nerve root avulsion [19]. Secondly, the images may contain motion artifacts; as 
Addar et al. Medical Imaging and Radiology 2014,

http://www.hoajonline.com/journals/pdf/2054-1945-2-1.pdf

doi: 10.7243/2054-1945-2-1

with all kinds of MRI, these artifacts decrease the quality of images obtained [19]. In the case of large meningoceles, some nerve roots may not be clearly visualized because of the lesions; therefore, the nerve roots that are invisible cannot be deemed normal or injured [18]. Finally, the difficulty in determining the exact level of injury and the inclusion of vertebral arteries and the spinal venous plexus in myelographic images may disturb the image and the interpretation obtained [26]. Considering the aforementioned flaws, MRM is not the method of choice for traumatic injuries and cannot replace CTM as the gold standard since the latter can help overcome these obstacles [18].

\section{Magnetic resonance neurography (MRN)}

MRN is a special type of MRI that is tissue specific and capable of eliciting the morphological features of nerves, such as their caliber, continuity, and relation to nearby structures such as nerves, muscles and bones, as well as pathological features of the nerves(e.g., nerve fibrosis, inflammation, and edema) (Figure 7). The term MRN is used when the imaging is done for the peripheral nerves, while in the case of the central nervous system, the terms "tractography" and "diffuse tensor imaging" are used [27].

The diagnostic efficacy of MRN is high. Filler found that more than $96 \%$ of MRN examinations resulted in either a specific finding in the nerves involved or a clear definitive statement that the nerves of interest are normal in appearance. MRN depends on the alterations in endoneural fluid content in the nerves since pathological processes increase this fluid relative to other cellular components. MRN findings include disruptions of the course of the proximal elements at the scalene triangle, fibrous band entrapments affecting the C8 and T1 spinal nerves and the lowertrunk of the brachial plexus, gross distortions of the mid-plexus, hyperintensity consistent with nerve irritation at the level of the first rib, and distal plexus hyperintensity. Notably, three-dimensional (3D) reconstructions of the MRN images yield more information than the two dimensional (2D) images by $28 \%$, and this is greatest with the brachial plexus; therefore, 3D reconstructions are considered an essential part of diagnostic interpretations [27]. Du et al., [28] showed that MRN provided more diagnostic information than electrodiagnostic studies and concluded that MRN is best used when MRI and electrodiagnostic studies are unavailing regarding spinal or peripheral nerve pathology, if MRI shows multilevel disease and electro-diagnostic studies are unable to confirm these results, and in patients who are unable to undergo electrodiagnostic studies (i.e., patients on anti-coagulants or a coexistent disease that reduces accuracy such as diabetes) [29].

In addition, MRN can localize trauma, radiation injury, and neoplasms of the brachial plexus and peripheral nerves. An important aspect of MRN is that it is most useful when the onset of symptoms is less than 1 year and is less useful when it has been more than 2 years. Also to be noted is that MRN

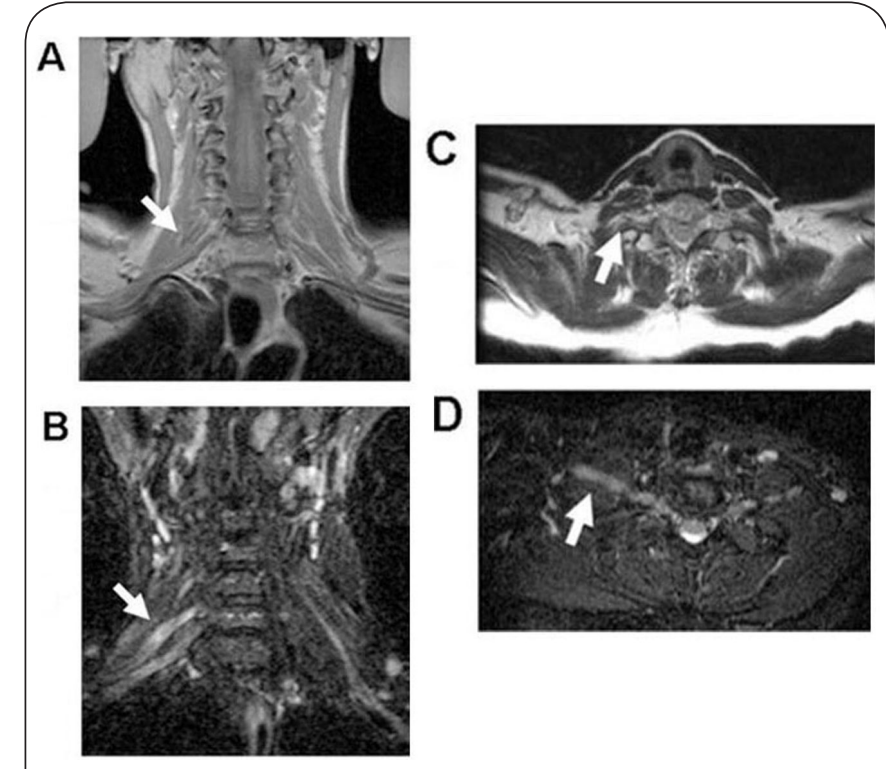

Figure 7. Images obtained in a 62-year-old woman with a metastatic tumor to brachial plexus and a history of metastatic breast cancer. After a course of chemotherapy, the patient noted right index finger numbness that progressed to full arm numbness and paresis of the biceps brachii and wrist movement. Routine coronal (A) and axial (C) MR images failed to reveal abnormalities. Coronal (B) and axial (D) STIR images through the brachial plexus, however, showed abnormal increased T2 signal in the right C- 6 and C-7 roots (arrows). The patient underwent exploration for biopsy, which confirmed infiltrating ductal breast carcinoma within the epineurium and perineurium. Intraoperatively, the nerves looked grossly normal and selection of the biopsy site relied entirely on MRN imaging. Reprinted from the Journal of Neurosurgery, 112, Rose Du, M.D., Ph.D., Kurtis I. Auguste, M.D., Cynthia T. Chin, M.D., John W. Engstrom, M.D., and Philip R. Weinstein, M.D., 362-371, 2010 with permission from the Journal of Neurosurgery.

can detect pathologies in a specific anatomical location (brachial plexus and peripheral nerves), but not in different locations at the same time [28]. Ordering errors are mainly due to the fact that many referring physicians are not familiar with nerve imaging [27].

\section{Computed tomography with myelography (CTM)}

CTM is the current gold standard for imaging avulsion injuries to the brachial plexus $[30,18]$. The diagnostic accuracy of CTM is equal to or greater than that of standard myelography and MRI [30]. Carvalho et al., found that the preoperative diagnostic accuracy of CTM is $85 \%$, where as that of MRI is $52 \%$ [23]. Notably, Abul-kasim et al., recently demonstrated that the accuracy of MRI is $88 \%$, with a sensitivity of $90 \%$ and specificity of $87 \%$; however, this study was conducted on 7 patients and was retrospective in nature [24]. Axial slices to visualize the brachial plexus should extend between $\mathrm{C} 4$ and $\mathrm{T} 1$ and are taken parallel to the cervical discs. Three-millimeter slices can demonstrate both ventral and dorsal rootlets on both sides 
when evaluating intradural cervical roots. CTM misdiagnosis occurs mainly in the setting of traumatic meningoceles and intradural fibrosis; to avoid this, 1-millimeter slices should be used when traumatic meningoceles are present, and in the case of intradural fibrosis, only surgical exploration will provide an accurate assessment of the cervical roots status. In some cases wherein CTM does not demonstrate the nerve roots, the patient should undergo a hemi-laminectomy to determine the status of the cervical roots [23]. CTM is also useful in evaluating tumors infiltrating the brachial plexus, since it is superlative in the detection of bony erosions of the spine as well as changes in the neural foramina [31]. Obstetric brachial plexus lesions (OBPL) can be evaluated by CTM [32]. Steens et al., [33] found that in 58\% of the patients selected for surgery for OBPL, preoperative CTM showed root avulsions. In $20 \%$ of the patients, root avulsion was also found at levels that were not expected on the basis of clinical examination. One of the important points to be considered for applying CTM in evaluating OBPL is that the numbering of the levels must be done accurately; this can be achieved by obtaining both coronal and sagittal multi-planar reconstructions. The authors concluded that all preoperative patients with obstetric brachial plexus lesions should undergo CTM, although several investigators, including Al-Qattan [32], have questioned the use of CTM for a considerable amount of time since CTM is invasive and requires sedation of the child. Nevertheless, currently, CTM is strongly recommended for any patient undergoing reconstruction for OBPL.

\section{Sonography of the brachial plexus}

Sonography does not have the same quality as MRI in evaluating soft tissues, such as the brachial plexus; however, there are many advantages of sonography, making it an important complimentary tool in imaging the brachial plexus. The first and most important advantage is patient satisfaction. Middleton et al., [34] evaluated 118 patients having shoulder pain with both MRI and sonography and found that patients preferred sonography 10 times to MRI. Reasons for this include the time consumed in evaluation, since $25 \%$ of the patients perceived MRI as more lengthy, where as less than $2 \%$ found sonography more lengthy. Another reason is the interactive nature of sonography compared to MRI, which puts the patients to ease during the evaluation. Other advantages include the ability to conduct an ultrasound examination in virtually any patient, ability to perform real-time examination, availability of important information about the adjacent blood vessels through Doppler sonography, the ability to differentiate fluid and solid material better than MRI, ability to guide therapeutic interventions, ability to provide bilateral comparison, and availability of a more flexible field of view [35]. In addition, sonography costs much less than MRI and utilizes no radiation, which is a major advantage over most imaging modalities.

Almost all pathologies affecting the brachial plexus can be visualized or at least screened for through sonography. Entrapment neuropathies due to a cervical rib, elongated C7 transverse process, and other causes of the thoracic outlet syndrome can also be detected. This is of paramount importance in children since it minimizes the radiation risk from standard radiographs [36]. Sonography is also valuable in the detection of nerve tumors from thebrachial plexus. Although certain features may aid in distinguishing benign lesions from malignant ones, it is not possible to determine this based on sonography alone. Sonography is also useful in guiding interventions (i.e., biopsy of a tumor and brachial plexus anesthesia) and in the postoperative followup of cases (Figure 8) [37-38].
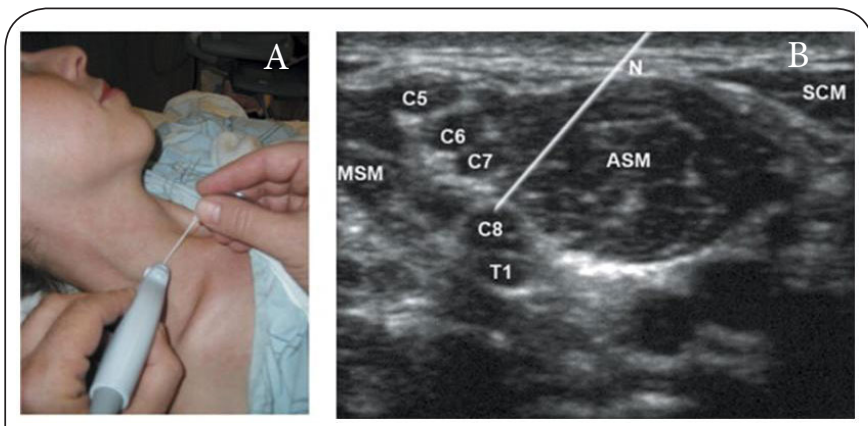

Figure 8. Ultrasound-guided interscalene block. (A) In this example, the needle is introduced from an antero-medial to postero-lateral direction, in-plane with the transducer, which is held in an axial oblique orientation. (B) Ultrasound scan of real-time needle guidance during interscalene block.

$\mathrm{N}=$ needle (enhanced with photo editing for emphasis); C5$\mathrm{T} 1=$ cervical and first thoracic nerve roots;

ASM=anterior scalene muscle; $\mathrm{MSM}=$ middle scalene muscle; SCM=sternocleidomastoid muscle. Reprinted from Seminars in Anesthesia, Perioperative Medicine and Pain, 26(4), Steven L. Orebaugh, Paul Bigeleisen, 180-188, 2007 with permission from ELSEVIER.

In the case of traumatic brachial plexus injuries (pre- and post-ganglionic) sonography can detect root avulsion, nerve injuryin the form of a neuroma, and scar tissue formation [39]. Chen et al., [40] found that sonography can characterize pre- and post-ganglionic lesions preoperatively, although their detection is dependent on the experience level of the technician. Detection of nerve root injuries is also very high, since it has been reported to be $100 \%$ for $\mathrm{C} 5$ to $\mathrm{C} 7$ as well as for the upper and middle trunks, $84 \%$ for $\mathrm{C} 8$ and the lower trunk, and $64 \%$ for $\mathrm{T} 1$, in all subjects.

Follow up of brachial plexus injuries is a major area in which sonography is useful since it helps in monitoring the progress of lesions, such as tumors and traumatic nerve lesions [41]. The brachial plexus appears as a hypoechoic structure under sonography. To assess the pathological lesions in the supraand infra-clavicular regions, it is best to use an axial oblique plane running parallel to the subclavian artery. In the case 
Addar et al. Medical Imaging and Radiology 2014,

http://www.hoajonline.com/journals/pdf/2054-1945-2-1.pdf

doi: 10.7243/2054-1945-2-1

of cervical root avulsions, a coronal oblique plane is most reliable and accurate. Intact roots appear as well delineated hypoechoic structures leaving the intervertebral foramina. Avulsions appear as empty neural foramina. Sonography is yet to be fully implemented in clinical practice; this is because experience and a good anatomical background are needed to conduct this test. The $\mathrm{C} 8$ and $\mathrm{T} 1$ nerve roots are difficult to evaluate since they are too caudal and deep. Finally, the roots originate from within the vertebral column and bone blocks the sonographic visibility; therefore, if there were an isolated intradural damage, it would not be visualized by sonography [39].

\section{Conclusion}

The brachial plexus is a complex component of the nervous system. Injury to the brachial plexus can affect the peripheral nervous system and, potentially, the central nervous system; this highlights the need for a deep knowledge of the modalities currently available for the evaluation of the brachial plexus. Traditional MRI is and should be, for the time being, the imaging method of choice for non-traumatic plexopathies. MRM should be used for traumatic injuries, such as traumatic meningoceles and root avulsions. MRN would be most beneficial when traditional MRI and electrodiagnostics are inconclusive in their results, as well as for determining whether tumors of the plexus are primary or secondary and for the surgical planning in patients with plexus trauma or lesions. CTM remains the gold standard for evaluating traumatic injuries of the brachial plexus although MRM is of much higher diagnostic accuracy; however, its accuracy is compromised in the case of large meningoceles and artifacts and CTM would be needed. Sonography is soon to become the stethoscope of this century, with the ability to visualize and screen for many pathologies all over the human body. In the brachial plexus, it can screen for most lesions and, therefore, it can be considered as a baseline or a screening investigation; however, it is hindered by the experience required by the technician and the complex anatomical location of the plexus, which highlight the need for developing new ultrasound transducers.

\section{Competing interests}

The authors declare that they have no competing interests.

\section{Authors' contributions}

\begin{tabular}{|l|c|c|}
\hline Authors' contributions & AMA & AAAS \\
\hline Research concept and design & $\checkmark$ & $\checkmark$ \\
\hline Collection and/or assembly of data & $\checkmark$ & $\checkmark$ \\
\hline Writing the article & $\checkmark$ & $\checkmark$ \\
\hline Critical revision of the article & $\checkmark$ & $\checkmark$ \\
\hline Final approval of article & $\checkmark$ & $\checkmark$ \\
\hline
\end{tabular}

\section{Acknowledgement}

We would like to thank Dr. Abdulmonem AlSiddiky for his constant support.
Publication history

Received: 02-Nov-2013 Revised: 22-Dec-2013

Accepted: 27-Jan-2014 Published: 10-Feb-2014

\section{References}

1. Midha R. Epidemiology of brachial plexus injuries in a multitrauma population. Neurosurgery. 1997; 40:1182-8. | Article | PubMed

2. Dorsi MJ, Hsu W and Belzberg AJ. Epidemiology of brachial plexus injury in the pediatric multitrauma population in the United States. J Neurosurg Pediatr. 2010; 5:573-7. | Article | PubMed

3. Benjamin K. Part 1. Injuries to the brachial plexus: mechanisms of injury and identification of risk factors. Adv Neonatal Care. 2005; 5:181-9. | Article I PubMed

4. Zapalowicz K, Radek A, Lyczak P, Blaszczyk B and Skiba P. [Brachial plexus tumors]. Neurol Neurochir Pol. 2002; 36:697-710. | PubMed

5. Saifuddin A. Imaging tumours of the brachial plexus. Skeletal Radiol. 2003; 32:375-87. | Article | PubMed

6. Jarmundowicz $\mathrm{W}$, Jablonski $\mathrm{P}$ and Zaluski R. [Brachial plexus tumors-neurosurgical treatment]. Neurol Neurochir Pol. 2002; 36:925-35. I PubMed

7. Kori SH, Foley KM and Posner JB. Brachial plexus lesions in patients with cancer: 100 cases. Neurology. 1981; 31:45-50. | Article | PubMed

8. Fabian VA, Wood B, Crowley P and Kakulas BA. Herpes zoster brachial plexus neuritis. Clin Neuropathol. 1997; 16:61-4. | Article | PubMed

9. Suarez GA, Giannini C, Bosch EP, Barohn RJ, Wodak J, Ebeling P, Anderson $\mathrm{R}$, McKeever PE, Bromberg $\mathrm{MB}$ and Dyck PJ. Immune brachial plexus neuropathy: suggestive evidence for an inflammatory-immune pathogenesis. Neurology. 1996; 46:559-61. | Article | PubMed

10. Sanders RJ, Hammond SL and Rao NM. Diagnosis of thoracic outlet syndrome. J Vasc Surg. 2007; 46:601-4. | Article | PubMed

11. Hamburger V. Development of the nervous system. Ann $N$ Y Acad Sci. 1952; 55:117-32. | Article | PubMed

12. Johnson EO, Vekris M, Demesticha T and Soucacos PN. Neuroanatomy of the brachial plexus: normal and variant anatomy of its formation. Surg Radiol Anat. 2010; 32:291-7. | Article I PubMed

13. Leffert RD. Brachial-plexus injuries. N Engl J Med. 1974; 291:1059-67. | Article I PubMed

14. Bertelli JA and Ghizoni MF. Concepts of nerve regeneration and repair applied to brachial plexus reconstruction. Microsurgery. 2006; 26:23044. | Article | PubMed

15. Martinoli C, Gandolfo N, Perez MM, Klauser A, Palmieri F, Padua L and Tagliafico A. Brachial plexus and nerves about the shoulder. Semin Musculoskelet Radiol. 2010; 14:523-46. I Article I PubMed

16. Kichari JR, Hussain SM, Den Hollander JC and Krestin GP. MR imaging of the brachial plexus: current imaging sequences, normal findings, and findings in a spectrum of focal lesions with MR-pathologic correlation. Curr Probl Diagn Radiol. 2003; 32:88-101. | Article | PubMed

17. Tagliafico A, Succio G, Serafini $G$ and Martinoli C. Diagnostic accuracy of MRI in adults with suspect brachial plexus lesions: a multicentre retrospective study with surgical findings and clinical follow-up as reference standard. Eur J Radiol. 2012; 81:2666-72. | Article | PubMed

18. Yoshikawa T, Hayashi N, Yamamoto S, Tajiri Y, Yoshioka N, Masumoto T, Mori H, Abe O, Aoki S and Ohtomo K. Brachial plexus injury: clinical manifestations, conventional imaging findings, and the latest imaging techniques. Radiographics. 2006; 26 Suppl 1:S133-43. | Article | PubMed

19. Gasparotti R, Ferraresi S, Pinelli L, Crispino M, Pavia M, Bonetti M, Garozzo $D$, Manara $O$ and Chiesa A. Three-dimensional MR myelography of traumatic injuries of the brachial plexus. AJNR Am J Neuroradiol. 1997; 18:1733-42. | Article | PubMed

20. Li CS, Huang GS, Wu HD, Chen WT, Shih LS, Lii JM, Duh SJ, Chen RC, Tu HY and Chan WP. Differentiation of soft tissue benign and malignant peripheral nerve sheath tumors with magnetic resonance imaging. Clin Imaging. 2008; 32:121-7. | Article | PubMed

21. Iyer VR, Sanghvi DA and Merchant N. Malignant brachial plexopathy: A 
Addar et al. Medical Imaging and Radiology 2014,

pictorial essay of MRI findings. Indian J Radiol Imaging. 2010; 20:274-8. I Article | PubMed Abstract | PubMed Full Text

22. Wittenberg KH and Adkins MC. MR imaging of nontraumatic brachial plexopathies: frequency and spectrum of findings. Radiographics. 2000 20:1023-32. | Article | PubMed

23. Carvalho GA, Nikkhah G, Matthies C, Penkert G and Samii M. Diagnosis of root avulsions in traumatic brachial plexus injuries: value of computerized tomography myelography and magnetic resonance imaging. J Neurosurg. 1997; 86:69-76. | Article | PubMed

24. Abul-Kasim K, Backman C, Bjorkman A and Dahlin LB. Advanced radiological work-up as an adjunct to decision in early reconstructive surgery in brachial plexus injuries. I Brachial Plex Peripher Nerve Inj. 2010; 5:14. | Article | PubMed Abstract | PubMed Full Text

25. Nakamura T, Yabe $Y$, Horiuchi $Y$ and Takayama $S$. Magnetic resonance myelography in brachial plexus injury. J Bone Joint Surg Br. 1997; 79:7649. | Article | PubMed

26. El Mogy SA, Mazroa JA, KassemM and El Mogy MS. Role of FIESTA combined with conventional MRI in the evaluation of traumatic brachial plexus roots injury. The Egyptian Journal of Radiology and Nuclear Medicine. 2011; 42:57-67. | Article

27. Filler A. Magnetic resonance neurography and diffusion tensor imaging: origins, history, and clinical impact of the first 50,000 cases with an assessment of efficacy and utility in a prospective 5000-patient study group. Neurosurgery. 2009; 65:A29-43. | Article | PubMed Abstract | PubMed Full Text

28. Du R, Auguste KI, Chin CT, Engstrom JW and Weinstein PR. Magnetic resonance neurography for the evaluation of peripheral nerve, brachial plexus, and nerve root disorders. J Neurosurg. 2010; 112:362-71. | Article I PubMed

29. Chemali KR and Tsao B. Electrodiagnostic testing of nerves and muscles: when, why, and how to order. Cleve Clin J Med. 2005; 72:37-48. | Article I PubMed

30. Giuffre JL, Kakar S, Bishop AT, Spinner RJ and Shin AY. Current concepts of the treatment of adult brachial plexus injuries. J Hand Surg Am. 2010; 35:678-88. | Article | PubMed

31. Binder DK, Smith JS and Barbaro NM. Primary brachial plexus tumors: imaging, surgical, and pathological findings in $\mathbf{2 5}$ patients. Neurosurg Focus. 2004; 16:E11. | Pdf | PubMed

32. Al-Qattan MM. Obstetric brachial plexus injuries. J Hand SurgAm. 2003; 3:41-54.

33. Steens SC, Pondaag W, Malessy MJ and Verbist BM. Obstetric brachial plexus lesions: CT myelography. Radiology. 2011; 259:508-15. | Article PubMed

34. Middleton WD, Payne WT, Teefey SA, Hildebolt CF, Rubin DA and Yamaguchi K. Sonography and MRI of the shoulder: comparison of patient satisfaction. AJR Am J Roentgenol. 2004; 183:1449-52. | Article I PubMed

35. Nazarian LN. The top 10 reasons musculoskeletal sonography is an important complementary or alternative technique to MRI. AJR Am J Roentgenol. 2008; 190:1621-6. | Article | PubMed

36. Mangrulkar VH, Cohen $\mathrm{HL}$ and Dougherty $\mathrm{D}$. Sonography for diagnosis of cervical ribs in children. J Ultrasound Med. 2008; 27:1083-6. | Article | PubMed

37. Martinoli C, Bianchi S, Gandolfo N, Valle M, Simonetti S and Derchi LE. US of nerve entrapments in osteofibrous tunnels of the upper and lower limbs. Radiographics. 2000; 20 Spec No:S199-213. | Article | PubMed

38. Lee FC, Singh $\mathrm{H}$, Nazarian LN and Ratliff JK. High-resolution ultrasonography in the diagnosis and intraoperative management of peripheral nerve lesions. J Neurosurg. 2011; 114:206-11. | Article | PubMed

39. Haber HP, Sinis N, Haerle M and Schaller HE. Sonography of brachial plexus traction injuries. AJR Am J Roentgenol. 2006; 186:1787-91. । Article | PubMed

40. Chen DZ, Cong R, Zheng MJ, Zhu T, Coles G, Feng H, Zhou XD and Zhu YS. Differential diagnosis between pre- and postganglionic adult traumatic brachial plexus lesions by ultrasonography. Ultrasound Med Biol. 2011; 37:1196-203. | Article | PubMed
41. Padua L, Liotta G, Di Pasquale A, Granata G, Pazzaglia C, Caliandro P and Martinoli $C$. Contribution of ultrasound in the assessment of nerve diseases. Eur J Neurol. 2012; 19:47-54. | Article | PubMed

Citation:
Addar AM and Al-Sayed AA. Update and
review on the basics of brachial plexus
imaging. Med Imaging Radiol. 2014; $2: 1$.
http://dx.doi.org/10.7243/2054-1945-2-1

